\title{
Search for electroweak production of charginos and neutralinos in final states with one lepton, jets and missing transverse momentum with the ATLAS experiment
}

\author{
VU Ngoc Khanh ${ }^{a, *}$ on behalf of the ATLAS Collaboration \\ ${ }^{a}$ Aix Marseille Univ, CNRS/IN2P3, CPPM, Marseille, France \\ 163, Avenue de Luminy - Case 902 - 13288 Marseille cedex 09 \\ E-mail: nkvu@cppm.in2p3.fr
}

\begin{abstract}
This document presents a search for electroweak production of mass degenerate charginoneutralino pairs in the context of R-parity conserving supersymmetric simplified models in which the chargino decays into $\mathrm{W}$ boson and the lightest neutralino, while the next-to-lightest neutralino decays into either Higgs or $Z$ boson, in addition to the lightest neutralino. This search concentrates on final states characterized by the presence of one isolated charged lepton (either electron or muon) accompanied by jets and missing transverse momentum. The analysis exploits an integrated luminosity of $139 \mathrm{fb}^{-1}$ which corresponds to the full Run-2 of proton-proton collisions data recorded by the ATLAS detector at Large Hadron Collider. No statistically significant evidence of a deviation from the Standard Model expectation is observed. Expected and observed 95\% CL limits are set based on the chargino and the lightest neutralino mass, assuming pure wino production cross-sections.
\end{abstract}

The Ninth Annual Conference on Large Hadron Collider Physics - LHCP2021

7-12 June 2021

Online

${ }^{*}$ Speaker 


\section{Introduction}

This document presents a search for direct production of a chargino-neutralino pair $\left(\tilde{\chi}_{1}^{ \pm} \tilde{\chi}_{2}^{0}\right)$, where the $\tilde{\chi}_{1}^{ \pm}$subsequently decays into a $W$ boson and a $\tilde{\chi}_{1}^{0}\left(\tilde{\chi}_{1}^{ \pm} \rightarrow W \tilde{\chi}_{1}^{0}\right)$, and the $\tilde{\chi}_{2}^{0}$ into a Higgs boson and a $\tilde{\chi}_{1}^{0}\left(\tilde{\chi}_{2}^{0} \rightarrow h \tilde{\chi}_{1}^{0}\right)$. The search targets a $W$ boson which decays into an electron or muon (and corresponding neutrino) and a Higgs boson which decays into a pair of $b$-quarks, as shown in Figure 1. This search is considered in the context of $R$-parity conserving supersymmetric simplified model where the $\tilde{\chi}_{1}^{0}$ is the lightest supersymmetric particle (LSP), being stable, weakly interacting and thus invisible to the detector; the $\tilde{\chi}_{1}^{ \pm}$and $\tilde{\chi}_{2}^{0}$ are nearly mass degenerate; the branching ratios of $\tilde{\chi}_{1}^{ \pm} \rightarrow W \tilde{\chi}_{1}^{0}$ and $\tilde{\chi}_{2}^{0} \rightarrow h \tilde{\chi}_{1}^{0}$ are assumed to be $100 \%$ and the branching ratio of $h \rightarrow b \bar{b}$ is taken to be

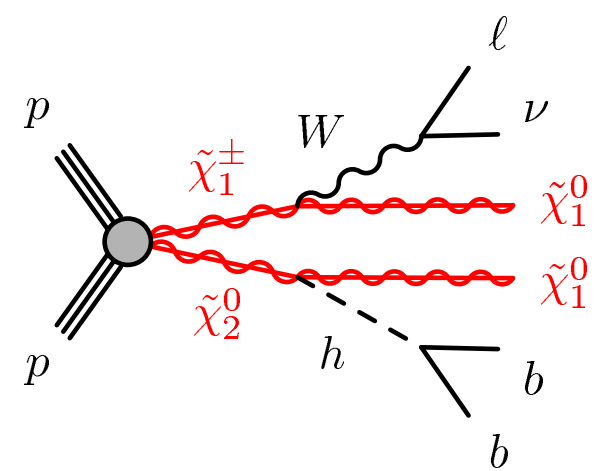

Figure 1: A diagram illustrating the signal scenario considered for the production of a chargino and a next-to-lightest neutralino. $58.3 \%$ as expected for the SM Higgs boson. The signature consists of exactly one light lepton ( $e$ or $\mu$ ), two $b$-jets, and large missing transverse momentum $\left(\mathbf{p}_{\mathrm{T}}^{\mathrm{miss}}\right)$ from neutralinos and neutrinos. The search for charginos and neutralinos via the Higgs boson targets an integrated luminosity of $139 \mathrm{fb}^{-1}$, corresponding to the full Run 2 data collected at the ATLAS detector. Previous search for the same final state but using $36.1 \mathrm{fb}^{-1}$ of data has been reported in Ref [1] by the ATLAS collaboration.

\section{Analysis strategy}

Events are recorded with the lowest-threshold missing transverse momentum trigger [2] with the offline requirement of $E_{\mathrm{T}}^{\mathrm{miss}}>240 \mathrm{GeV}$, where $E_{\mathrm{T}}^{\text {miss }}$ is defined as the magnitude of the negative vectorial sum of the transverse momenta of the reconstructed objects in the final state. To target the signal topology, events are required to have exactly one lepton, two or three jets, two of which must be $b$-tagged. Furthermore, the invariant mass of the two $b$-jets, $m_{b \bar{b}}$, is required to pass $100<m_{b \bar{b}}<140$ $\mathrm{GeV}$ in order to preferentially select $b$-jets from the Higgs boson decays.

Three separate classes of signal regions (SRs), labelled as SRLM, SRMM and SRHM are further defined with the transverse mass, $m_{\mathrm{T}}=$

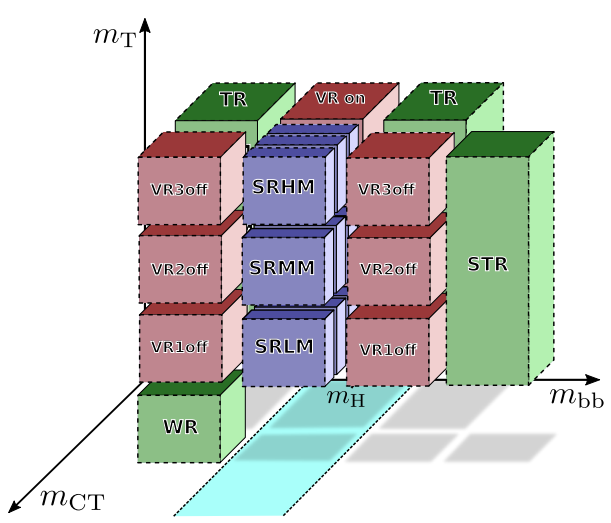

Figure 2: Illustration of all regions of interest used in the analysis. $\sqrt{2 p_{\mathrm{T}}^{l} E_{\mathrm{T}}^{\text {miss }}\left(1-\cos \left[\Delta \phi\left(\mathbf{p}_{\mathrm{T}}^{l}, \mathbf{p}_{\mathrm{T}}^{\text {miss }}\right)\right]\right)}$, required to be in the ranges of $[100,160],[160,240]$ and $>240 \mathrm{GeV}$ to target signal models with low, medium and high mass-splittings $\Delta m\left(\tilde{\chi}_{1}^{ \pm} / \tilde{\chi}_{2}^{0}, \tilde{\chi}_{1}^{0}\right)=$ $m\left(\tilde{\chi}_{1}^{ \pm} / \tilde{\chi}_{2}^{0}\right)-m\left(\tilde{\chi}_{1}^{0}\right)$, respectively. Additionally, SRHM also requires the invariant mass of the lepton and the leading $b$-jet, $m\left(l, b_{1}\right)$, to be larger than $120 \mathrm{GeV}$ in order to further suppress 
$t \bar{t}$ and single-top background events. In order to estimate the expected yields of the dominant backgrounds ( $t \bar{t}$, single-top and $W+$ jets) in the SRs, a set of orthogonal control regions (CRs) is defined for each of these backgrounds harmonizing cuts on $m_{\mathrm{T}}, m_{b \bar{b}}$ and the contransverse mass $m_{\mathrm{CT}}=\sqrt{2 p_{\mathrm{T}}^{b_{1}} p_{\mathrm{T}}^{b_{2}}\left(1+\cos \Delta \phi_{b b}\right)}$. Finally, two sets of orthogonal validation regions (VRs) are defined for each SR, including off-peak $\left(m_{b \bar{b}}<100\right.$ or $\left.m_{b \bar{b}}>140 \mathrm{GeV}\right)$ and on-peak $m_{b \bar{b}}$ regions. The extrapolation from the CRs to the SRs is checked in these VRs. A schematic view for the design of the SRs, CRs and VRs is shown in Figure 2.

Model-dependent exclusion limits at 95\% confidence level (CL) are obtained with each SR binned in three separate $m_{\mathrm{CT}}$ regions of [180,230], [230, 280] and $>280 \mathrm{GeV}$, thus providing nine bins in total for a simultaneous two-dimensional fit in $m_{\mathrm{CT}}$ and $m_{\mathrm{T}}$. For model-independent limits, the $m_{\mathrm{CT}}$ bins of each SR are merged and the upper bounds on $m_{\mathrm{T}}$ for SRLM and SRMM are removed.

\section{Systematic uncertainties}

The systematic uncertainties are evaluated for all the simulated signal and background samples. For uncertainties on theoretical modelling of the backgrounds in the SRs, the dominant uncertainty sources are the $t \bar{t}$ parton shower in SRLM (10\%), and the single-top generator uncertainties in SRMM (10\%) and SRHM (21\%). The overall theoretical uncertainties for SUSY signals in the SRs range from about $10 \%$ in the region with a large splitting between the $\tilde{\chi}_{1}^{ \pm} / \tilde{\chi}_{2}^{0}$ and $\tilde{\chi}_{1}^{0}$ masses to about $25 \%$ in the mass spectra with small mass splitting. The dominant detector systematic effects are the uncertainties associated with the jet energy scale (JES) and jet energy resolution (JER), the $E_{\mathrm{T}}^{\text {miss }}$ modelling, and pile-up. They contribute 5-10\% in the SRs, having less significant impact than the theoretical ones. The MC statistical uncertainties are 5-18\% in the SRs.

\section{Results}

The background normalisation factors obtained from the background-only fit are $1.02_{-0.09}^{+0.07}$ for $t \bar{t}, 0.6_{-0.25}^{+0.5}$ for single-top, and $1.22_{-0.24}^{+0.26}$ for $W+$ jets. The compatibility of the observed and expected event yields in all the analysis regions is illustrated in Figure 3. No significant excess over the SM prediction is observed in data. The 95\% CL upper limits, p-values and the significances obtained from the model-independent fit as well as the observed CL of the background-only hypothesis are shown in Table 1. Model-dependent exclusion limits at 95\% CL of this analysis as well as the other analyses, which also search for the same production via the Higgs boson, are shown as a function of the masses of the supersymmetric particles in Figures 4 . The observed limit in our analysis extends up to about $740 \mathrm{GeV}$ in $m\left(\tilde{\chi}_{1}^{ \pm} / \tilde{\chi}_{2}^{0}\right)$ for massless $\tilde{\chi}_{1}^{0}$. This improves on the previous limit by about $200 \mathrm{GeV}$ due to the increase of the integrated luminosity and the improved two-dimensional fit. The limits obtained is the strongest one at the low mass-splitting regions among all the searches for this electroweakino production. 


\begin{tabular}{lcccccc}
\hline Signal Region & $\langle\epsilon \sigma\rangle_{\text {obs }}^{95}[\mathrm{fb}]$ & $S_{\text {obs }}^{95}$ & $S_{\text {exp }}^{95}$ & $\mathrm{CL}_{\mathrm{B}}$ & $p_{0}$ & $\mathrm{Z}$ \\
\hline SRLM (disc.) & 0.26 & 36.8 & $20.0_{-5.4}^{+8.0}$ & 0.97 & 0.03 & 1.88 \\
SRMM (disc.) & 0.18 & 24.8 & $15.3_{-4.6}^{+6.2}$ & 0.94 & 0.06 & 1.54 \\
SRHM (disc.) & 0.11 & 14.7 & $9.7_{-2.7}^{+3.3}$ & 0.89 & 0.10 & 1.30 \\
\hline
\end{tabular}

Table 1: Left to right: 95\% CL upper limits on the visible cross-section and on the number of signal events. The third column shows the expected $95 \%$ CL upper limit (and its $\pm 1 \sigma$ excursions) on the number of signal events if no BSM signal is present. The last three columns indicate the $\mathrm{CL}_{B}$ value, i.e. the confidence level observed for the background-only hypothesis, the discovery p-value $\left(p_{0}\right)$ and the significance $Z$ [3].

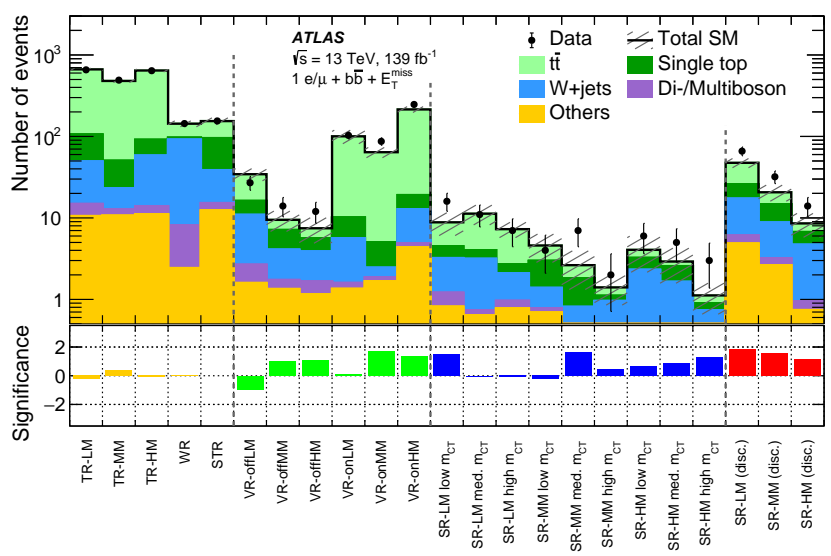

Figure 3: Comparison of the observed and expected event yields in control, validation, exclusion, and discovery signal regions. Uncertainties in the background estimates include both the statistical (in the simulated event yields) and systematic uncertainties. The bottom panel shows the significance [3] of the differences between the observed and expected yields. Not all regions shown here are statistically independent.

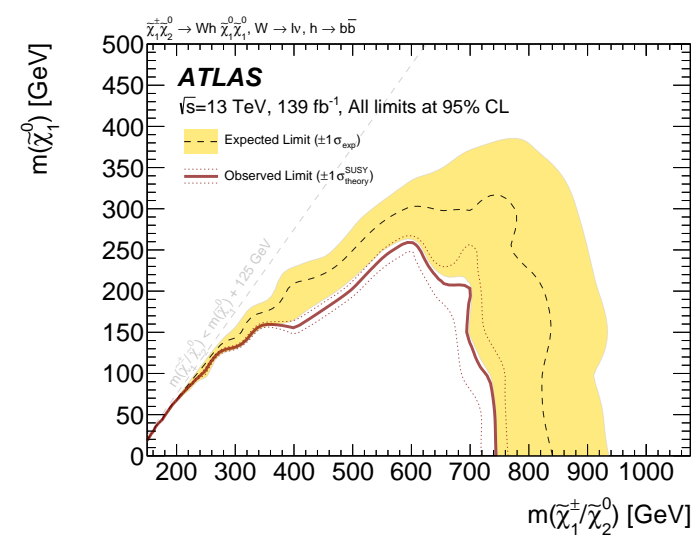

(a)

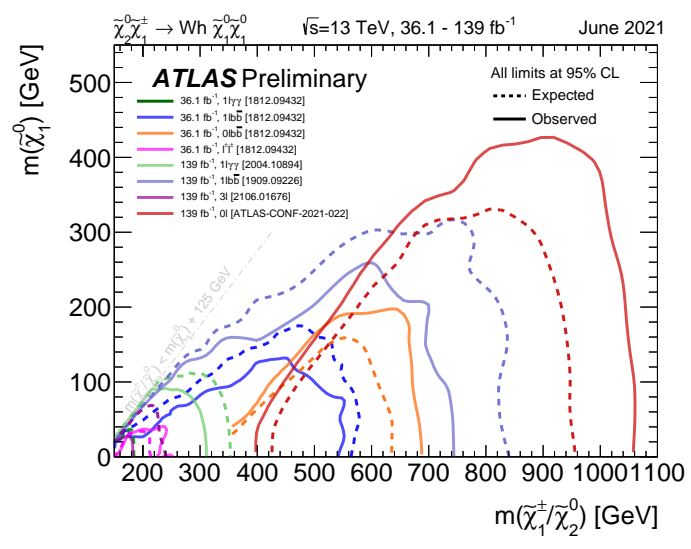

(b)

Figure 4: Model-dependent exclusion contour at 95\% CL on the production of a chargino and a next-tolightest neutralino. 4a shows only the results obtained from this analysis, while $4 \mathrm{~b}$ shows additionally results from other analyses that also search for the same electroweakino production. 


\section{References}

[1] M. Aaboud, G. Aad, B. Abbott, O. Abdinov, B. Abeloos, D. Abhayasinghe et al., Search for chargino and neutralino production in final states with a higgs boson and missing transverse momentum at $\sqrt{s}=13$ tev with the atlas detector, Physical Review D 100 (2019) .

[2] G. Aad, B. Abbott, D.C. Abbott, A. Abed Abud, K. Abeling, D.K. Abhayasinghe et al., Performance of the missing transverse momentum triggers for the atlas detector during run-2 data taking, Journal of High Energy Physics 2020 (2020) .

[3] R.D. Cousins, J.T. Linnemann and J. Tucker, Evaluation of three methods for calculating statistical significance when incorporating a systematic uncertainty into a test of the background-only hypothesis for a poisson process, Nuclear Instruments and Methods in Physics Research Section A: Accelerators, Spectrometers, Detectors and Associated Equipment 595 (2008) 480-501. 